\title{
PROPRIEDADES DE CHAPAS FABRICADAS COM PARTÍCULAS DE MADEIRA DE Eucalyptus urophylla S. T. Blake E DE Schizolobium amazonicum Herb. ${ }^{1}$
}

Rafael Baptista Naumann ${ }^{2}$, Benedito Rocha Vital ${ }^{3}$, Angêlica de Cássia Oliveira Carneiro ${ }^{3}$,Ricardo Marius Della Lucia ${ }^{3}$, José de Castro Silva ${ }^{3}$, Ana Márcia Macêdo Ladeira Carvalho ${ }^{3}$ e Andréia Colli ${ }^{2}$

\begin{abstract}
RESUMO - Este trabalho teve como objetivo avaliar as propriedades de chapas de madeira aglomerada fabricadas com partículas de Eucalyptus urophylla (massa específica $=0,55 \mathrm{~g} / \mathrm{cm}^{3}$ ) e de Schizolobium amazonicum (Paricá) (massa específica $=0,30 \mathrm{~g} / \mathrm{cm}^{3}$ ). Foram confeccionadas chapas com cinco proporções de madeira e dois tipos de partículas (maravalhas e cavacos). As chapas apresentaram dimensões de $60 \mathrm{~cm}$ x $60 \mathrm{~cm} \mathrm{x} 1 \mathrm{~cm}$ e massa específica média de $0,60 \mathrm{~g} / \mathrm{cm}^{3}$. Utilizou-se adesivo à base de uréia-formaldeído, na proporção de $8 \%$. As chapas foram prensadas à temperatura de $170^{\circ} \mathrm{C}$ e $3,2 \mathrm{MPa}$ de pressão, em ciclos de $8 \mathrm{~min}$, e as suas propriedades foram determinadas segundo a norma NBR 14810-3. A massa específica, a dureza Janka e a expansão linear não foram influenciadas pelas variáveis experimentais. De modo geral, o aumento na porcentagem de paricá elevou a resistência à flexão, ao arrancamento de parafuso e à tração perpendicular. $\mathrm{O}$ tipo de partícula afetou significativamente apenas a resistência à tração perpendicular e o inchamento em espessura. As chapas produzidas com partículas provenientes de cavacos (coeficiente de esbeltez menor) tiveram maior resistência à tração perpendicular. Contudo, apresentaram valores mais elevados de inchamento em espessura.
\end{abstract}

Palavras-chave: Aglomerado, Eucalyptus urophylla e Schyzolobium amazonicum.

\section{PROPERTIES OF FOILS MANUFACTURED WITH PARTICLES OF WOOD OF Eucalyptus urophylla S. T. Blake AND OF Schizolobium amazonicum Herb.}

\begin{abstract}
This work aimed to evaluate the properties of particleboards produced with Eucalyptus urophylla $\left(\right.$ density $0.55 \mathrm{~g} / \mathrm{cm}^{3}$ ) and Schizolobium amazonicum (density $0.30 \mathrm{~g} / \mathrm{cm}^{3}$ ). Five wood proportions and two types of particle (shavings and chips) were used to manufacture the boards. Board dimension was $60 \mathrm{~cm}$ $x 60 \mathrm{~cm} x 1 \mathrm{~cm}$ and average density $0.60 \mathrm{~g} / \mathrm{cm}^{3}$. Eight percent of urea-formaldehyde adhesive was used. Boards were pressed at $170^{\circ} \mathrm{C}$ and $32 \mathrm{kgf} / \mathrm{cm}^{2}$ of pressure in 8 minute cycles. Board properties were determined according to NBR 14810-3 standard. There was no influence of the variables on board density and linear expansion. Generally, increasing the percentage of paricá increased the values of some properties such as MOE, MOR, screw holding, water absorption after 24 hours and internal bond, probably due to increased compaction rate. The type of particle used affected only the internal bond and thickness swelling of the boards. The boards produced with wood chip-originated particles (smaller slenderness ratio) presented higher internal bond. However, thickness swelling was higher.
\end{abstract}

Keywords: Particleboards, Eucalyptus urophylla and Schyzolobium amazonicum.

\footnotetext{
${ }^{1}$ Recebido em 29.05.2007 e aceito para publicação em 22.08.2008.

${ }^{2}$ Programa de Pós-Graduação em Ciência Florestal da Universidade Federal de Viçosa (UFV). E-mail: <naumann@ gmail.com>.

${ }^{3}$ Departamento de Engenharia Florestal da UFV. E-mail: <bvital@ ufv.br>.
} 


\section{INTRODUÇÃO}

Aglomerado é um painel manufaturado com partículas de madeira ou de outro material ligno-celulósico unidas com um adesivo.

O segmento produtor de painéis de madeira, especialmente de MDF e de aglomerados, tem demonstrado elevado dinamismo, como reflexo das altas taxas de crescimento da indústria moveleira - a principal consumidora de painéis.

De acordo com a Revista da Madeira (2003), o Brasil dispõe de condições favoráveis para se tornar um importante produtor mundial de painéis de madeira, uma vez que detém tecnologia que permite a utilização de extensos plantios de árvores de rápido crescimento, além da base florestal nativa, ainda muito pouco explorada. Essa característica, associada ao dinamismo do mercado interno e internacional, tem sido um dos principais alavancadores dos novos investimentos.

A maioria dos processos tecnológicos empregados pelas indústrias de produtos florestais possui rígidas exigências em relação à espécie, ao tamanho das partículas e à qualidade da matéria-prima. Além disso, a demanda crescente por madeira de qualidade e que consiga suprir as necessidades do mercado vem fazendo com que cresça a idéia de utilização de espécies alternativas de reflorestamento.

De acordo com Geimer e Price (1978), muitos fatores influenciam as propriedades de chapas de flocos e de aglomerado. Algumas variáveis do processo, como a velocidade de fechamento da prensa, a umidade do colchão, a massa específica da chapa, o tipo, a quantidade e a qualidade do adesivo, o tempo e a temperatura de prensagem interagem entre si ou com algumas características da matéria-prima, como o tipo da madeira utilizada, a massa específica da madeira, a geometria das partículas, o pH da madeira e outros. Essas interações podem ocorrer simultaneamente e, às vezes, de forma não linear, favorecendo ou prejudicando algumas propriedades das chapas.

Segundo Michaque, citado por Peixoto e Brito (2000), a geometria das partículas é um dos principais fatores a ser levado em consideração na produção das chapas de madeira aglomerada. Alterada a geometria das partículas, é necessário ajustar outras variáveis do processo, como a quantidade de adesivo.
Segundo Maloney (1989), a resistência e rigidez à flexão e a resistência à tração paralela e perpendicular à superfície, ao arrancamento de parafusos e pregos são influenciadas pelo tamanho das partículas que, além disso, influenciam a absorção de água e de outros líquidos que causam alteração na estabilidade dimensional e modificações superficiais nas chapas.

Segundo Vital et al. (1992), partículas longas e finas produzem chapas com maior resistência à flexão estática e maior estabilidade dimensional, enquanto chapas fabricadas com partículas curtas e espessas aumentam a sua resistência à tração perpendicular ou ligação interna.

De acordo com Kelly (1977), outro fator de grande importância, senão o mais importante, para a qualidade final das chapas, se refere à massa específica da madeira e à taxa de compactação do colchão, ou seja, a relação entre a massa específica final do painel e a massa específica da madeira. Quanto maior a compactação, maior o contato entre partículas e entre partículas e adesivo. Dessa forma, podem-se ter painéis com a mesma massa específica final, porém com taxas de compactação diferentes, em função da massa específica da madeira.

Segundo Kawai e Sassaki, citados por Iwakiri et al. (2000), as propriedades mecânicas dos painéis de madeira aglomerada apresentam relação direta e linear com a razão de compactação.

Hillig et al. (2002) concluíram que as propriedades mecânicas da chapa, como o módulo de ruptura (MOR), o módulo de elasticidade (MOE) e a ligação interna (LI) são afetadas pela massa específica da madeira utilizada. Gouveia et al. (2000) afirmaram que a utilização da madeira de Eucalyptus urophylla com idade superior a 18 anos é desaconselhável na fabricação de chapas, em virtude de sua alta massa específica.

Moslemi (1974) observou que painéis produzidos com espécies de baixa massa específica geralmente apresentaram maior resistência à flexão e à tração, melhor módulo de elasticidade e melhor ligação interna do que painéis obtidos de espécies de alta massa específica, para chapas de igual massa específica.

Em relação à mistura de espécies, Vital (1973) concluiu que a mistura de madeiras de diferentes massas específicas se apresenta como alternativa para o aproveitamento de madeiras com maiores massa específicas, resultando em chapas com massa específica e propriedades aceitáveis 
pelo mercado. Essa mistura de espécies pode representar uma saída para o aproveitamento de madeiras tropicais, de maiores e menores massas específicas. Assim, o paricá pode ser uma espécie promissora porque apresenta massa específica baixa, o que permite o ajuste de sua mistura com outras espécies de maior massa específica.

De acordo com Hillig et al. (2002), de modo geral, misturando espécies com diversas massas específicas, pose-se obter um valor médio que beneficie as propriedades das chapas. Assim, o objetivo geral deste trabalho foi avaliar as propriedades das chapas de madeira aglomerada, utilizando madeiras de Eucalyptus urophylla e Schizolobium amazonicum, em diferentes proporções e tipos de partículas. Entre os objetivos específicos, destacam-se:

1 - Avaliar as propriedades físicas e mecânicas das chapas de madeira aglomerada fabricadas com partículas de Eucalyptus urophylla e de Schizolobium amazonicum.

2 - Avaliar o efeito da mistura das madeiras de Eucalyptus urophylla e Schizolobium amazonicum, nas propriedades das chapas de aglomerado.

\section{MATERIAL E MÉTODOS}

Foram utilizadas madeiras de Eucalyptus urophylla com massa específica de $0,55 \mathrm{~g} / \mathrm{cm}^{3}$, proveniente de plantios pertencentes à CAF, no Município de Três Marias (região do Cerrado de Minas Gerais), e de Schizolobium amazonicum com massa específica de $0,30 \mathrm{~g} / \mathrm{cm}^{3}$, também conhecido como paricá, proveniente do Município de Dom Eliseu, no Estado do Pará.

Após o desdobro da madeira em tábuas, parte das tábuas foi processada em plainas para a geração das maravalhas, e outra parte foi processada em picador para gerar cavacos de madeira. Posteriormente, tanto as maravalhas quanto os cavacos foram reduzidos em moinho-de-martelo. Após a classificação para remoção de "finos" (partículas que passaram em peneira de malha de 2,0 $\mathrm{mm} \times 2,0 \mathrm{~mm}$ ) e acondicionamento em estufa à temperatura de $53 \pm 2{ }^{\circ} \mathrm{C}$, obtiveram-se partículas com aproximadamente $3 \%$ de umidade.

Para determinação do coeficiente de esbeltez, foram medidas as espessuras e comprimentos de aproximadamente 100 partículas.

Para fabricação das chapas, as partículas foram encoladas com adesivo uréia-formaldeído, com teor de sólidos de $65 \%$, na razão de $8 \%$, em relação à massa das partículas, em tambor rotatório dotado de uma pistola automática, acionada por compressor de ar. As chapas foram produzidas com massa específica nominal de 0,60 $\mathrm{g} / \mathrm{cm}^{3}$ e dimensões de $60 \mathrm{~cm}$ x $60 \mathrm{~cm}$ x $1 \mathrm{~cm}$. A prensagem ocorreu a uma temperatura de $170^{\circ} \mathrm{C}$, pressão de $3,2 \mathrm{MPa}$ e tempo de prensagem de $8 \mathrm{~min}$. Foram estabelecidos 10 tratamentos, divididos em dois tipos diferentes de partículas, que se diferenciavam pelas dimensões, e cinco diferentes composições de chapas para cada tipo de partícula, como mostrado no Quadro 1.

Após a estabilização das chapas em temperatura ambiente, elas foram seccionadas para retirada de corposde-prova, que foram climatizados em uma câmara climática a uma umidade de $65 \pm 5 \%$ e temperatura de $20 \pm 3{ }^{\circ} \mathrm{C}$. Os ensaios foram realizados de acordo com os procedimentos descritos na norma NBR 14810-3 (ABNT, 2002). Os resultados foram avaliados através de análises de variância. Quando se observaram diferenças significativas, as médias foram comparadas entre si, pelo teste de Tukey a $95 \%$ de probabilidade estatística.

Quadro 1 - Tratamentos experimentais

Table 1 - Experimental treatments

\begin{tabular}{cccc}
\hline Tratamentos & Taxa de compactação & Composição das chapas & Tipo de partícula \\
\hline T 1 & 1,09 & $100 \%$ eucalipto / 0\% paricá & PPM* \\
T 2 & 1,23 & $75 \%$ eucalipto / 25\% paricá & PPM \\
T 3 & 1,41 & $50 \%$ eucalipto / 50\% paricá & PPM \\
T 4 & 1,65 & $25 \%$ eucalipto / 75\% paricá & PPM \\
T 5 & 2,00 & $0 \%$ eucalipto / 100\% paricá & PPM \\
T 6 & 1,09 & $100 \%$ eucalipto / 0\% paricá & PPC \\
T 7 & 1,23 & $75 \%$ eucalipto / 25\% paricá & PPC \\
T 8 & 1,41 & $50 \%$ eucalipto / 50\% paricá & PPC \\
T 9 & 1,65 & $25 \%$ eucalipto / 75\% paricá & PPC \\
T10 & 2,00 & $0 \%$ eucalipto / 100\% paricá & \\
\hline
\end{tabular}

*Em que: PPM = partículas geradas a partir de maravalhas; $\mathrm{PPC}=$ partículas geradas a partir de cavacos. 


\section{RESULTADOS E DISCUSSÕES}

Os coeficientes de esbeltez são mostrados no Quadro 2 e os valores médios das propriedades físicas, no Quadro 3. Observa-se, nesses quadros, que o coeficiente de esbeltez das partículas fabricadas com maravalhas foi maior que o das partículas fabricadas a partir de cavacos.

A massa específica média foi de $0,57 \mathrm{~g} / \mathrm{cm}^{3}$, o que as classifica, segundo a normaANSI/A1-280-93, na categoria de baixa massa específica (menor do que $640 \mathrm{~kg} / \mathrm{m}^{3}$ ). Não se verificou diferença significativa entre as massas específicas das chapas, indicando que houve controle adequado do experimento.

A expansão linear, uma das mais importantes propriedades físicas das chapas, uma vez que caracteriza o comportamento do material mediante a variação da umidade relativa, também não foi influenciada pelas variáveis em estudo. A média foi de $0,46 \%$, excedendo, contudo, o máximo estabelecido na norma ANSI/A1-280-93, que é de $0,35 \%$ para qualquer tipo de chapa. Entretanto, podese observar, exceto para as chapas fabricadas com $25 \%$ de partículas de eucalipto e $75 \%$ de partículas de paricá, oriundas de maravalhas, tendência de diminuição da expansão linear à medida que se eleva a concentração de paricá nas chapas, ou seja, aumenta-se a taxa de compactação. O aumento da taxa de compactação é responsável pela formação de uma chapa mais homogênea e com maior massa específica. Esse aumento faz com que se diminuam os vazios entre as partículas. Vale ressaltar que não foi aplicada parafina às partículas, o que contribuiu para o aumento da adsorção de água e de expansão linear. Apesar de ser superior ao valor mínimo estabelecido na norma ANSI/A1-280-93, esses resultados são similares aos encontrados por Macnatt (1974) para diversos tipos de chapas de aglomerado.

A absorção de água durante as duas primeiras horas de imersão também não foi influenciada pela composição das chapas ou pelas dimensões das partículas. A média de todos os tratamentos foi de $99,8 \%$, valor superior ao encontrado por Batista et al. (2007). Isso pode ser devido ao uso do adesivo à base de uréia-formaldeído, que é solúvel em água. Já o ensaio de absorção, após 24 h de imersão, foi influenciado pela composição das chapas, conforme mostrado na Figura 1. Observou-se que a absorção de água aumentou à medida que aumentou também o porcentual de paricá na composição das chapas. Isso pode dever-se à maior área superficial das partículas decorrentes da menor massa específica da madeira de paricá. As normas pertinentes da ABNT e ANSI não estabelecem valores máximos e mínimos de absorção de água por imersão para a comercialização de painéis de madeira. As médias de absorção de água foram inferiores aos valores encontrados por Batista et al. (2007).

$\mathrm{O}$ inchamento em espessura, conforme mostrado na Figura 2, foi influenciado pelo tipo de partícula utilizada, porém não se verificou influência significativa da composição das chapas na propriedade em questão. Após $2 \mathrm{~h}$ de imersão em água, o inchamento médio das chapas fabricadas com partículas provenientes de maravalhas (PPM) foi de 14,4\%, enquanto o inchamento para partículas provenientes de cavacos (PPC) foi de 19\%. O incremento no inchamento em espessura após $24 \mathrm{~h}$ de imersão foi de $17,30 \%$ nas chapas fabricadas com PPM e de $22,4 \%$ naquelas com PPC. Quando são comparados os tipos de partículas, percebeu-se que as PPM apresentam um coeficiente de esbeltez maior, apresentando, nesse caso, espessura menor em relação às PPC. Apesar do aumento da eficiência da utilização do adesivo à medida que utilizam partículas mais espessas, fato esse verificado por Moslemi (1974), as chapas produzidas com partículas provenientes de cavacos (PPM) apresentaram maior inchamento em espessura. Esse fato, também verificado por Cabral (2005), pode ser explicado pelo melhor ajuste das partículas mais finas e esbeltas durante a formação do colchão e da prensagem. A compactação diminui a quantidade de vazios entre as partículas, dificultando a entrada da umidade nos sítios de adsorção. Todos os valores obtidos para inchamento em espessura ultrapassaram o máximo aceito pela norma ANSI A 208.1, que estabelece o máximo de $0,35 \%$ para essa categoria de chapas.

Quadro 2 - Coeficiente de esbeltez de cada espécie e cada tipo de partícula Table 2 - Slenderness ratio for each species and type of particle used

\begin{tabular}{lccc}
\hline Espécie & Tipo de partícula & Espessura & Comprimento \\
\hline Eucalipto & PPM & 0,278 & 6,87 \\
Eucalipto & PPC & 0,708 & 8,36 \\
Paricá & PPM & 0,493 & 7,89 \\
Paricá & PPC & 0,851 & 7,51 \\
\hline
\end{tabular}

Em que: $\mathrm{PPM}=$ partículas geradas a partir de maravalhas; $\mathrm{PPC}=$ partículas geradas a partir de cavacos. 
Quadro 3 - Valores médios das propriedades físicas dos painéis Table 3 - Mean physical property values of the boards

\begin{tabular}{ccccccccc}
\hline Tratamentos & Partículas & TU & ME & EL & I2h & I24h & A2h & A24h \\
\hline T 1 & PPM & 10,4 & 631,1 & 0,56 & 13,8 & 16,8 & 89,2 & 97,2 \\
T 2 & PPM & 10,7 & 581,2 & 0,49 & 15,5 & 18,6 & 106,5 & 105,5 \\
T 3 & PPM & 11,0 & 538,5 & 0,40 & 11,5 & 14,2 & 95,8 & 105,2 \\
T 4 & PPM & 10,3 & 579,6 & 0,50 & 16,8 & 19,7 & 109,3 & 119,9 \\
T 5 & PPM & 9,7 & 543,2 & 0,33 & 14,5 & 17,1 & 98,9 & 117,9 \\
T 6 & PPC & 10,6 & 566,8 & 0,61 & 18,6 & 21,8 & 87,8 & 101,9 \\
T 7 & PPC & 11,8 & 623,9 & 0,47 & 20,2 & 23,3 & 93,2 & 100,2 \\
T 8 & PPC & 10,7 & 604,3 & 0,47 & 18,5 & 20,8 & 98,4 & 106,5 \\
T 9 & PPC & 9,8 & 541,4 & 0,38 & 19,2 & 23,0 & 110,9 & 126,5 \\
T10 & PPC & 10,0 & 532,2 & 0,43 & 18,7 & 23,1 & 108,4 & 127,0 \\
\hline
\end{tabular}

Em que: PPM = partículas geradas a partir de maravalhas; $\mathrm{PPC}=$ partículas geradas a partir de cavacos; TU = teor de umidade $(\%)$; ME = massa específica $(\mathrm{kg} / \mathrm{m} 3) ; \mathrm{EL}=$ expansão linear $(\%) ; \mathrm{I} 2$ h e I24 = Inchamento em espessura (\%) após 2 e 24 h de imersão em água, respectivamente; A2 h e A24 h = absorção de água (\%) após 2 h e 24 h de imersão em água, respectivamente.

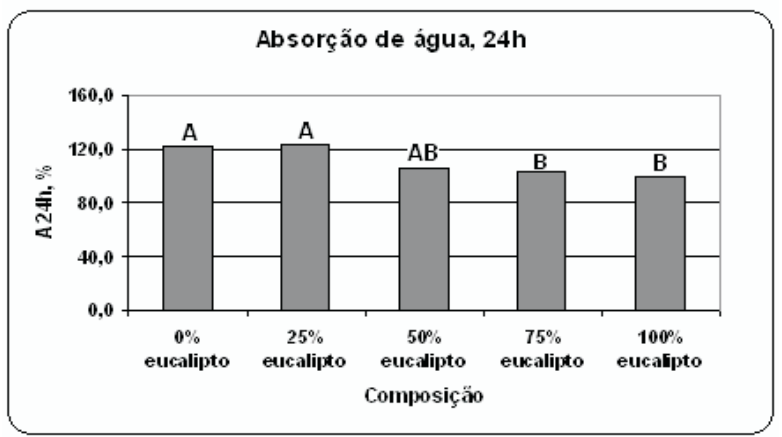

Figura 1 - Valores médios de absorção (\%) após 24 h de imersão em água (Médias seguidas pela mesma letra não diferem estatisticamente entre si, a $95 \%$ de probabilidade).

Figure 1 - Mean absorption values (\%) after 24 h of immersion in water.

As médias das propriedades mecânicas das chapas podem ser observadas no Quadro 4.

Apesar da grande amplitude de variação (149,5 a 394,20 kgf) na dureza Janka, a análise de variância não indicou diferenças significativas entre as chapas. Analisando os dados, observou-se que as variações ocorreram ao acaso dentro do mesmo tratamento. Isso ocorreu, provavelmente, porque o teste é realizado em uma pequena área, sendo afetado pela distribuição das partículas no local da inserção da semi-esfera. $\mathrm{O}$ valor médio de dureza Janka em todos os tratamentos foi de 255,8 kgf. Contudo, como ocorreu com a expansão linear, de modo geral, houve tendência de aumento nos valores de dureza à medida que se adicionou mais partícula de paricá. Essa variação pode ser devido ao aumento na taxa de compactação, associado com as dimensões das partículas. No caso das chapas produzidas com PPM, o aumento da taxa de compactação resultou numa chapa com maior massa específica básica e homogênea, ou seja, com menos vazios no seu interior.

De modo geral, observou-se aumento significativo nos valores de tração perpendicular, conforme mostrado na Figura 3, à medida que se aumentou a quantidade de paricá nas chapas, exceto nas chapas produzidas com $50 \%$ de partículas de paricá. Isso provavelmente se deva ao aumento na taxa de compactação das chapas, pois, de acordo com Kollmann et al. (1975), madeiras com massas específicas mais baixas são mais maleáveis e mais fáceis de comprimir. Em geral, são necessárias taxas de compactação elevadas, quando se trabalha com matéria-prima com massa específica mais baixa. Com isso, tem-se uma chapa com maior massa específica básica e mais homogênea.

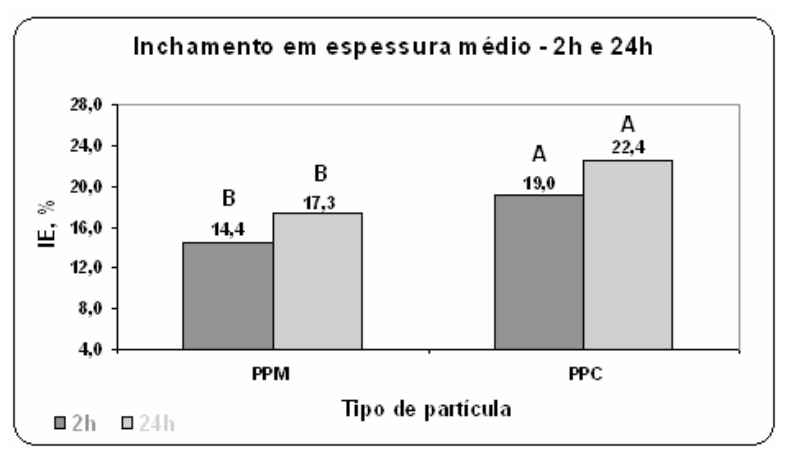

Figura 2 - Valores médios de inchamento em espessura (\%), após 2 e 24 h de imersão em água, em cada tipo de partícula (Médias seguidas pela mesma letra não diferem estatisticamente entre si, a $95 \%$ de probabilidade).

Figure 2 - Mean thickness swelling values (\%) after $2 \mathrm{~h}$ and 24 of immersion in water for each type of particle.

R. Árvore, Viçosa-MG, v.32, n.6, p.1143-1150, 2008 
Quadro 4 - Valores médios das propriedades mecânicas dos painéis Table 4-Mean mechanical property values of the boards

\begin{tabular}{|c|c|c|c|c|c|c|}
\hline Tratamentos & Partículas & ТP Мpa & MOR Mpa & MOE Mpa & AP N & DJ $\mathrm{N}$ \\
\hline $\mathrm{T} 1$ & PPM & 0,16 & 4,42 & 734,1 & 3,7 & 16,9 \\
\hline $\mathrm{T} 2$ & PPM & 0,18 & 5,13 & 696,8 & 4,6 & 22,7 \\
\hline T 3 & PPM & 0,42 & 10,09 & 1282,4 & 6,8 & 27,9 \\
\hline $\mathrm{T} 4$ & PPM & 0,31 & 8,71 & 1128,2 & 4,9 & 23,4 \\
\hline $\mathrm{T} 5$ & PPM & 0,46 & 13,96 & 1873,4 & 7,1 & 33,2 \\
\hline T 6 & PPC & 0,22 & 4,26 & 726,3 & 4,7 & 26,6 \\
\hline $\mathrm{T} 7$ & PPC & 0,44 & 7,27 & 970,6 & 5,3 & 27,8 \\
\hline $\mathrm{T} 8$ & PPC & 0,52 & 7,82 & 1052,2 & 6,0 & 25,0 \\
\hline T 9 & PPC & 0,35 & 8,44 & 1275,5 & 5,9 & 24,5 \\
\hline $\mathrm{T} 10$ & PPC & 0,58 & 9,83 & 1168,6 & 6,6 & 27,5 \\
\hline
\end{tabular}

Em que: $\mathrm{PPM}=$ partículas geradas a partir de maravalhas; $\mathrm{PPC}=$ partículas geradas a partir de cavacos. TP = tração perpendicular; $\mathrm{MOR}=$ módulo de ruptura em flexão; MOE = módulo de elasticidade em flexão; AP = resistência ao arrancamento de parafuso; e DJ = dureza Janka.

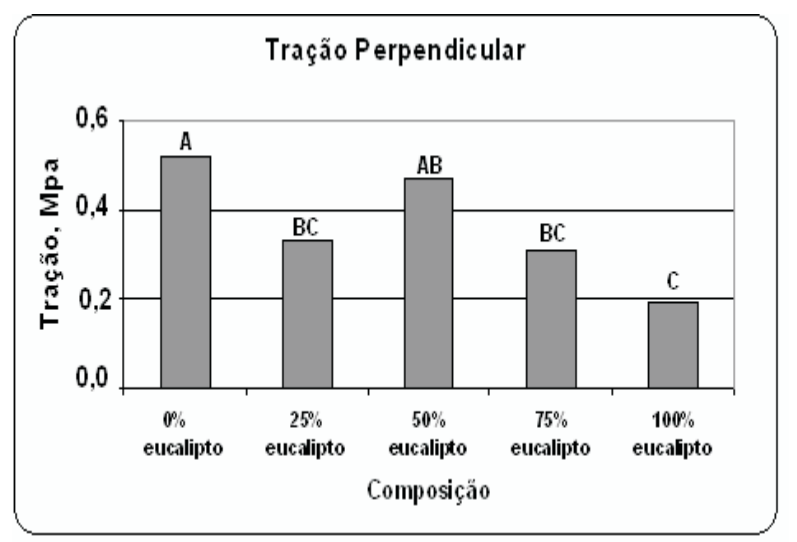

Figura 3 - Valores médios de tração perpendicular (Mpa) para cada composição das chapas (Médias seguidas pela mesma letra não diferem estatisticamente entre si, a $95 \%$ de probabilidade.

Figure 3-Mean internal bond values of (Mpa) for each board composition.

De modo geral, os valores médios de tração perpendicular das partículas provenientes de maravalhas (PPM) foram de 0,3 MPa, enquanto os valores médios das chapas produzidas com partículas provenientes de cavacos (PPC) foram de 0,42 MPa. De acordo com Vital et al. (1992), partículas mais espessas melhoram as propriedades de tração perpendicular, enquanto segundo Hse, citado por Kelly (1977), partículas maiores respondem melhor à alta compactação. De acordo com os autores citados, partículas com maiores dimensões aumentam a eficiência do adesivo utilizado. Isso ocorre porque, para uma mesma massa de partículas, aquelas com maiores dimensões possuem menor área superficial e recebem maior quantidade de adesivo por unidade de área. As resistências à tração perpendicular de todas as chapas foram superiores ao valor mínimo estabelecido na norma ANSI A 208.1(0,10 a 0,15 Mpa)

A resistência ao arrancamento de parafuso foi significativamente afetada pela composição das chapas, conforme pode ser observado na Figura 4. É provável que a redução dos vazios entre as partículas devido ao aumento na taxa de compactação, decorrente do aumento do percentual de partículas de paricá, tenha aumentado a compactação do material, resultando numa melhora nos valores dessa propriedade. Com exceção das chapas produzidas com $100 \%$ de partículas de eucalipto e com partículas provenientes de maravalhas (PPM), todas as outras apresentaram valores de AP compatíveis com os valores estabelecidos para comercialização desse tipo de chapa.

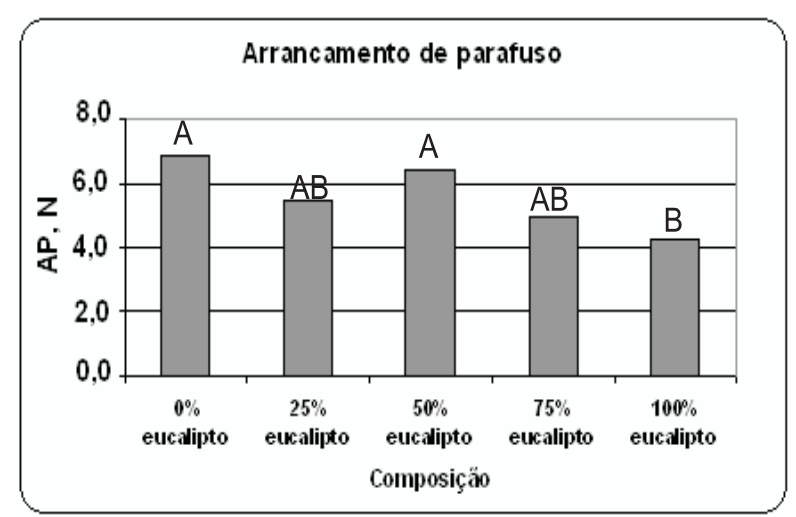

Figura 4 - Valores médios de arrancamento de parafuso (N) para cada composição das chapas (Médias seguidas pela mesma letra não diferem estatisticamente entre si, a $95 \%$ de probabilidade).

Figure 4-Mean nail holding $(N)$ values for each board composition. 
O módulo de ruptura, bem como o módulo de elasticidade, não foram afetados pelo tipo de partículas. Contudo, foi observado aumento nos valores dessas propriedades quando se utilizaram partículas com maior coeficiente de esbeltez. Segundo Lehmann (1974), ocorreu diminuição nos valores de MOR com o aumento da espessura das partículas quando todas as outras variáveis do processo foram mantidas constantes. As variações nos valores médios do MOE foram similares ao ocorrido com o MOR. De acordo com Geimer e Price (1978), as propriedades de flexão estática das chapas aumentam diretamente com o comprimento das partículas, devido às características geométricas das próprias partículas e sua capacidade em suportar esforços de flexão. A composição das chapas afetou, de forma significativa, a resistência à flexão estática, conforme-se pode observar nas Figuras 5 e 6 . Isso provavelmente ocorreu porque, para manter a massa específica das chapas constante, foi necessário elevar a taxa de compactação devido à menor massa específica da madeira de paricá. Com isso, à medida que adicionou partículas de paricá, aumentou-se a compactação das chapas e elevou, conseqüentemente, o MOE. Os valores de MOR e MOE situaram-se acima daqueles estabelecidos nas normas para comercialização desse tipo de produto.

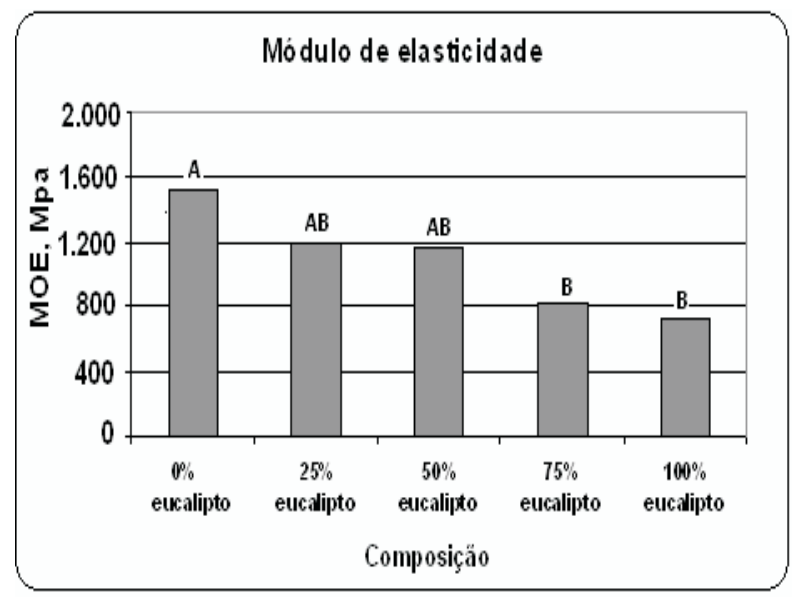

Figura 5 - Valores médios de MOE (Mpa) (Médias seguidas pela mesma letra não diferem estatisticamente entre si, a $95 \%$ de probabilidade).

Figure 5 - MOE mean values (Mpa).

\section{CONCLUSÕES}

A utilização de um porcentual maior de partículas de paricá, aliado ao aumento da taxa de compactação, melhorou a estabilidade dimensional das chapas e resultou em maior resistência mecânica.

Pode-se ajustar a massa específica das chapas misturando partículas de paricá com as de eucalipto.

Podem-se adequar as propriedades das chapas, alternado a geometria das partículas.

\section{RECOMENDAÇÕES}

Os resultados deste trabalho correspondem a um trabalho parcial, em que nem todas as variáveis do processo foram avaliadas. Além das variáveis estudadas, é necessário determinar o efeito da mistura de partículas de paricá com outras espécies que apresentem potencial econômico.

O efeito da variação na massa específica das chapas, da quantidade e qualidade do adesivo e da geometria das partículas e outras variáveis precisam ser determinadas.

Uma possível utilização comercial da madeira de Schizolobuim amazonicum (paricá) para a produção de chapas depende de análise econômica e de disponibilidade, a fim de se evitar futuros problemas de abastecimento.

\section{REFERÊNCIAS}

AMERICAN NATIONAL STANDARD - ANS. Mat-formed wood particleboard: Specification ANSI/A 208.1. 1993. Gaithersburg: National Particleboard Association, 1993. 9p.

ASSOCIAÇÃO BRASILEIRA DE NORMAS TÉCNICAS - ABNT. Chapas de madeira aglomerada - métodos de ensaio. ABNT. NBR 14810-3. Março, 2002.

BATISTA, D. C. et al. Fabricação de aglomerado de três camadas com madeira de Pinus elliottii Engelm. e casca de Eucalyptus pellita F. Muel. Cerne, v.13, n.2, p.178-187, 2007.

CABRAL, C. P. T. Propriedades de chapas tipo aglomerado e OSB, fabricadas com partículas e flocos de madeira de Eucalyptus grandis, Eucalyptus urophylla, Eucalyptus cloeziana e Pinus elliottii. 2005. 92 f. Dissertação (Mestrado em Ciência Florestal) Universidade Federal de Viçosa, Viçosa, MG, 2005.

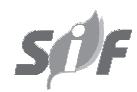

R. Árvore, Viçosa-MG, v.32, n.6, p.1143-1150, 2008 
GEIMER, R. L.; PRICE, E. W. Construction variables considered in the fabrication of structural flakeboard. In: STRUCTURAL FLAKEBOARD FROM FOREST RESIDUES: SYMPOSIUM PRESENTED BY THE USDA FOREST SERVICE, 1978, Kansas City. Proceedings... Washington: Forest Service. Departament of Agriculture, 1978. p.69-80.

GOUVEIA, N. F.; SANTANA, M. A. E.; SOUZA, M. R. Utilização da madeira de Eucalyptus grandis W. Hill ex. Maiden e Eucalyptus urophylla S.T. Blake na fabricação de chapas de partículas orientadas (OSB) e não orientadas. Revista Árvore, v.24, n.1, p.7-12, 2000.

HILLIG, E.; HASELEIN, C. R.; SANTINI, E. J. Estabilidade dimensional de chapas aglomeradas estruturais (flakeboards) fabricadas com madeira de Pinus, eucalipto e acácia-negra. Scientia Forestalis, v.9, n.1, p.127-134, 2002.

IWAKIRI, S. et al. Resíduos de serrarias na produção de painéis de madeira aglomerada de eucalipto. Scientia Agrária, v.1, n.1-2, p.23-28, 2000.

KELLY, M. W. Critical literature review of relationships between processing parameters and physical properties of particleboards. Washington: USDA Forest Service, 1977. 66p. (General Technical Report. FPL-10)

KOLLMANN, F. F. P.; KUENZI, E. W.; STAM, A. J. Principles of wood science and technology. New York: Springer-Verlag, 1975. v.2.703p.

LEHMANN, W. F. Properties of structural particleboards. Forest Products Journal, v.24, n. 1, p.19-26, 1974.

R. Árvore, Viçosa-MG, v.32, n.6, p.1143-1150, 2008
MALONEY, T. M. Modern particleboard and dry-process fiberboard manufacturing. San Francisco: Miller Freeman Publications, 1989.

McNATt, J. D. Properties of particleboards at various humidity conditions. Washington: USDA Forest Service Research, 1974. 23p. (Paper FPL, 225).

MOSLEMI, A. A. Particleboards. Illinois, Southern Illinois University Press, 1974. v.2. 245 p.

PEIXOTO, G. L; BRITO, E. O. Avaliação da granulometria de partículas de Pinus taeda combinadas com adesivos comerciais para a fabricação de aglomerados. Floresta e Ambiente, v.7, n.1, p.60-67, 2000.

REVISTA DA MADEIRA. Processo produtivo de chapas de fibra de média densidade (MDF). Curitiba: 2003. Disponível em: <http://www.remade.com.br/ revista $/$ materia.php?edicao $=71 \& \mathrm{id}=330>$. Acesso em: 10 jan. 2007.

VITAL, B. R. Effects of species and panel densities on properties of hardwood particleboard. 1973. $111 \mathrm{f}$. These (Master of Science - Forestry) University of Wisconsin, Madison, 1973.

VITAL, B. R.; HASELEIN, C. R.; DELLA LUCIA, R. M. Efeito da geometria das partículas nas propriedades das chapas de madeira aglomerada de Eucalyptus grandis (Hill ex-Maiden). Revista Árvore, v.16, n.1, p.88-96, 1992. 